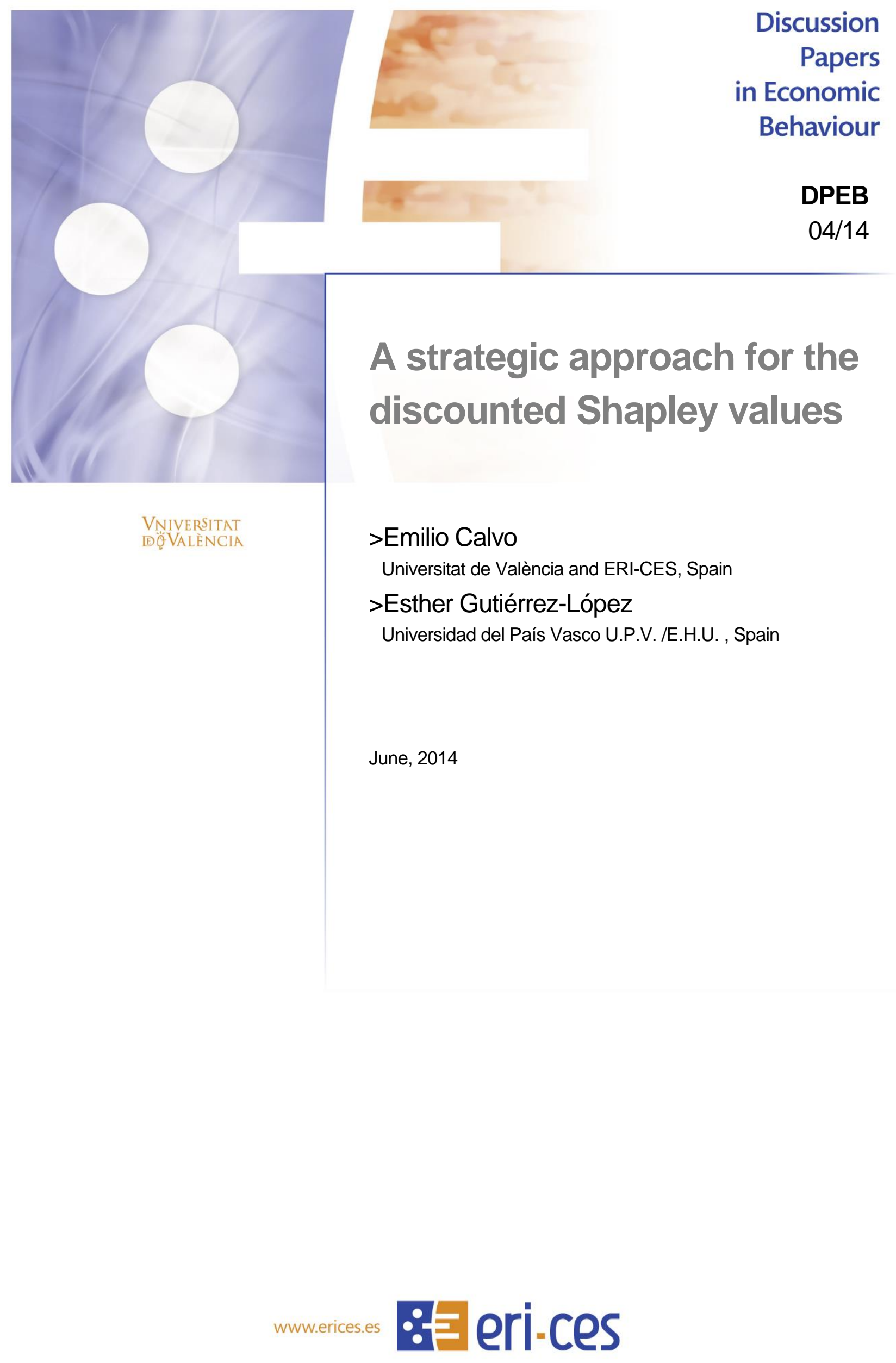




\title{
A strategic approach for the discounted Shapley values
}

\author{
Emilio Calvo*and Esther Gutiérrez-López ${ }^{\dagger}$
}

June 2014

\begin{abstract}
The family of discounted Shapley values is analyzed for cooperative games in coalitional form. We consider the bargaining protocol of the alternating random proposer introduced in Hart and Mas-Colell (1996). We demostrate that the discounted Shapley values arise as the expected payoffs associated with the bargaining equilibria when a time discount factor is considered. In a second model, we replace the time cost with the probability that the game ends without agreements. This model also implements these values in transferable utility games, moreover, the model implements the $\alpha$-consistent values in the nontransferable utility setting.
\end{abstract}

JEL Classification: C71.

KEYWORDS: Discounted Shapley value; egalitarianism; cooperative TU-games.

\section{Introduction}

In this note we consider the family of discounted Shapley values. These values were considered in Joosten (1996) and can be interpreted as a trade-off between marginalism and egalitarianism.

The Shapley value $(\varphi)$ is the reference rule for paying players according to their contribution in coalitional games with transferable utility (Shapley, 1953). Young's characterization of the Shapley value (Young, 1985) shows that it is the unique value that satisfies efficiency, symmetry and marginality. The last property says that if the marginal contributions of a player in two games are the same, then she should receive the same payoffs in both games. This property is a way of expressing the principle of paying players according with their productivity. An opposite option is to pay players equally, independent of their contributions in the game. This option is the egalitarian rule, expressed by the equal division solution $(E)$, that is, the equal division of the coalition's worth among all players. Recently, two different families have been considered that have both values as extreme cases: the egalitarian Shapley values $\beta^{\alpha}$, $\alpha \in[0,1]$, which is a convex combination between $\varphi$ and $E$, i.e. $\beta^{\alpha}=\alpha \varphi+(1-\alpha) E$; and the discounted Shapley values, $\varphi^{\alpha}, \alpha \in[0,1]$, where $\varphi^{1}=\varphi$ and $\varphi^{0}=E$.

\footnotetext{
*Departamento de Análisis Económico and ERI-CES. Universidad de Valencia. Avinguda dels Tarongers s/n. Edificio Departamental Oriental. 46022 Valencia. Spain. E-mail: Emilio.Calvo@uv.es

${ }^{\dagger}$ Departamento de Economía Aplicada IV. Universidad del País Vasco U.P.V./E.H.U., Spain. E-mail: mariaester.gutierrez@ehu.es
} 
In the Nash program two complementary approaches can support a solution: an axiomatic approach, which looks for the properties that characterize the solution, or a strategic approach, where the payoffs of the solution at hand arise as a result of the players' equilibrium behavior in a negotiation process. In the latter case, the interaction rules among players are given explicitly. This approach can help us to understand which factors are decisive in explaining why a particular cooperative solution concept should be taken into account.

In van den Brink and Funaki (2010), a bargaining game that implements the family of discounted Shapley values $\varphi^{\alpha}$ is offered. There, the bidding mechanism given by Pérez-Castrillo and Wettstein (2001), which implements the Shapley value, is considered. In the mechanism of Pérez-Castrillo and Wettstein, agents firstly bid for the right to be the proposer: every player $i$ announces a vector $\left(b_{j}^{i}\right)_{j \neq i}$ and the winner is the player who maximizes his relative willingness to be the proposer, i.e. $B^{i}=\sum_{j \neq i}\left(b_{j}^{i}-b_{i}^{j}\right)$. The winner has the right to make a proposal, $\left(y_{j}^{i}\right)_{j \neq i}$, which can be accepted or rejected. If it is accepted for all remaining players, each respondent $j$ receives $y_{j}^{i}+b_{j}^{i}$ and the proposer receives $v(N)-\sum_{j \neq i}\left(y_{j}^{i}+b_{j}^{i}\right)$. In the case where even one player rejects the proposal, the proposer leaves the game with $v(i)-\sum_{j \neq i} b_{j}^{i}$, the remaining agents $j \in N \backslash i$ continue the process with $b_{j}^{i}$ and bargain over $v(N \backslash i)$, and so on ${ }^{1}$. The way in which bids are performed guarantees that the payoffs associated with the subgame perfect equilibrium yield exactly the Shapley value in zero-monotonic games ${ }^{2}$. Van den Brink and Funaki (2010) introduce a parameter $\alpha \in[0,1]$ as a discount factor that determines the discounting of the available worth going from one round of negotiation to the next after a rejection of the proposal, i.e. the proposer $i$ leaves the game with $v(i)-\sum_{j \neq i} b_{j}^{i}$, and the remaining agents $j \in N \backslash i$ continue the process with $b_{j}^{i}$ and bargain over $\alpha v(N \backslash i)$, and so on. Alternatively, we can interpret $(1-\alpha)$ as a probability of breakdown in the case of rejection. Breakdown means that the bargaining is finished, leaving the proposer $i$ the final payoff $v(i)-\sum_{j \neq i} b_{j}^{i}$ and the respondents with $b_{j}^{i}, j \neq i$. With probability $\alpha$, the process continues to the next round with player set $N \backslash i$, and so on. This extended bidding mechanism implements the discounted Shapley value $\varphi^{\alpha}$.

In van den Brink, Funaki and Ju (2011) a variation of this discounted bidding mechanism is considered in which the discount is applied only in the first round of the negotiations. Alternatively, the possibility of the negotiations breaking down after rejection of the proposal occurs only in the first round. After that, the mechanism is the same as that of Pérez-Castrillo and Wettstein (2001). Hence, there is no possibility of breakdown anymore. With this variation in the discounted bidding mechanism the egalitarian Shapley value $\beta^{\alpha}$ is implemented.

Comparing both mechanisms, for the discounted Shapley values there is discounting (or probability of breakdown) in each of the $n$ possible rounds, whereas for the egalitarian Shapley values there is discounting (or probability of breakdown) only in the first round. From this point of view, the second mechanism exhibits a type of inconsistency between the first round and the remaining rounds. Therefore, the first mechanism and its corresponding associated solution, the discounted Shapley values, seems more natural.

\footnotetext{
${ }^{1}$ There are at most $n$ rounds.

${ }^{2} \mathrm{~A}$ game is zero-monotonic if $v(S) \leq v(T)$ whenever $S \subset T$, and $v(i)=0$ for all player $i$ in the game.
} 
In our paper we also follow the strategic approach. Our starting point is the bargaining procedure introduced by Hart and Mas-Colell (1996). There, a proposer is chosen randomly. He makes an offer, and the remaining players accept or reject the proposal. If all of the remaining players accept the proposal, the game ends. If a respondent rejects the offer, then, with a probability $\rho$, another proposer is selected randomly, and, with probability $(1-\rho)$, breakdown occurs: the proposer leaves the game with a payoff of zero, and the remaining players restart the bargaining process.

This procedure is a sequential, perfect information game, and it has a stationary subgame perfect equilibrium (in advance, SP equilibrium). The average of the equilibrium proposals is the Shapley value. Moreover, when the probability $\rho$ goes to one, the limit of these equilibrium proposals is the Shapley value.

In the same paper, Hart and Mas-Colell report a variant of this model that yields the egalitarian Shapley values. The variant applies only to what happens in the case of an offer's rejection: for a given parameter $0 \leq \alpha \leq 1$, if the offer is rejected, with probability $\rho$, the set of bargaining players is the same, with probability $(1-\rho) \alpha$, the proposer drops out of the game (receiving a payoff of zero), and with probability $(1-\rho)(1-\alpha) /(s-1)^{3}$ each respondent drops out of the game (receiving a payoff of zero). The average of the equilibrium proposals is now the egalitarian Shapley value $\beta^{\alpha}$, and when the probability $\rho$ goes to one, the limit of these equilibrium proposals is the average. In summary, the Shapley value appears only when the proposer drops out the game. In addition, the egalitarian value appears only when one of the respondents drops out. Finally, each egalitarian Shapley value $\beta^{\alpha}$ appears when there is a probability $\alpha$ that the proposer drops out and $(1-\alpha)$ that only one respondent drops out.

Immediately the question of whether the discounted Shapley values can be obtained with some suitable variation of the Hart and Mas-Colell model arises. Our paper answers this question. The result is simple: if we want to obtain a discounted Shapley value we need only to make the natural assumption that time is costly, which implies that players prefer to reach agreements at the beginning of bargaining. Assuming that all players have the same discount factor of $\delta \in[0,1]^{4}$, the main result is that when the risk of breakdown, $(1-\rho)$, and the discount factor, $\delta$, are considered simultaneously, the subgame perfect equilibria of the bargaining yields as average payoffs a discounted Shapley value of $\varphi^{\alpha}$, where

$$
\alpha=\frac{\delta(1-\rho)}{1-\delta \rho} .
$$

The intuition of this result is the following. The Shapley value is an expectation of the marginal contributions that a player makes to the coalitions to which he belongs. These players' marginal contributions arise from the computation of the equilibrium proposals because, when a player makes an offer, she has the opportunity to put the remaining players in front of an ultimatum situation: "if my offer is rejected, I may leave the game and you will lose my marginal contribution." The bargaining power is on the side of the proposer, and, hence, she is able to fully extract her expected marginal contributions in the game as the Shapley value does. Nevertheless, the time cost factor $\delta$ provides bargaining power to the respondent, because an offer's rejection forces the conclusion of an agreement at least to the next round, which lowers

\footnotetext{
${ }^{3}$ Where $s$ stands for the cardinality of the active coalition $S$ at this moment.

${ }^{4}$ That is, for every period $t=0,1,2 \ldots$, the utility of amount $x$ obtained at period $t$ can be represented by $\delta^{t} x$.
} 
the proposer's expected payoffs. Therefore, a higher cost of delay (lower $\delta$ ) diminishes the asymmetries between players attributable to the differences in their marginal contributions. This scenario raises the bargaining power of the respondent, and this condition is why the outcome will be more egalitarian.

In summary, the introduction of a discount factor yields a simple and natural explanation of why the family of discounted Shapley values should be considered.

A remarkable fact is that when the discount factor is considered these values are obtained only in their average. In the Hart and Mas-Colell case $(\delta=1)$ the average of the equilibrium proposals is always the Shapley value. When the fear of breakdown vanishes $(\rho \rightarrow 1)$, the equilibrium proposals converge to the average. The introduction of a discount factor $\delta$ now changes the convergence results drastically:

(i) The average of the equilibrium proposals is the discounted Shapley value.

(ii) With the discount factor $\delta<1$ fixed, when the fear of breakdown vanishes $(\rho \rightarrow 1)$ the average of the equilibrium payoffs converge to the equal division solution $\left(\alpha \rightarrow 0 \Rightarrow \varphi^{\alpha} \rightarrow E\right)$. Nevertheless, the proposals do not converge to the average.

(iii) With the risk of breakdown $(1-\rho)$ fixed, when the players are nearly fully patient in the bargaining $(\delta \rightarrow 1)$ the average of the equilibrium payoffs tend towards the Shapley value $\left(\alpha \rightarrow 1 \Rightarrow \varphi^{\alpha} \rightarrow \varphi\right)$. When the players are impatient $(\delta \rightarrow 0)$ the average of the equilibrium payoffs tend towards the equal division solution $\left(\alpha \rightarrow 0 \Rightarrow \varphi^{\alpha} \rightarrow E\right)$.

This lack of convergence of the proposals to their average is a serious handicap if we want to use the strategic approach to find a suitable definition of a discounted Shapley value in games without transferable utility. This scenario arises because even though the proposals belong to the boundary of the feasible utility set, it does not follow that their average belongs to the boundary (it will not be in the boundary if the feasible set is strictly convex). Hence, the limit of the average payoffs when $\rho \rightarrow 1$ need not be efficient.

For that reason we introduce an alternative variation of the Hart and Mas-Colell bargaining protocol. We replace the discount factor by a probability that either the proposer leaves the game, with a probability $(1-\rho) \alpha$, or the game ends without agreements, with a probability $(1-\rho)(1-\alpha)$, in the event that breakdown occurs. In TU-games this protocol also implements the discounted Shapley values. Moreover, the equilibrium proposals converge to their average when $\rho \rightarrow 1$. In hyperplane games ${ }^{5}$ these equilibrium payoffs converge to a unique payoff configuration, which is called the $\alpha$-consistent value of the hyperplane game. Assuming the same smoothness condition on the boundary of the feasible sets as in Hart and MasColell (1996), we can use the same convergence result that was used there to obtain now the $\alpha$-consistent values for the general setting of games without transferable utility.

The results obtained in this paper complement those obtained in van den Brink and Funaki (2010) in their study of the family of discounted Shapley values. We show that in the alternating randomproposer bargaining model of Hart and Mas-Colell, insofar as players have time preferences in the form of a constant discount rate, the discounted Shapley value immediately appears as the average equilibrium payoffs. In addition, we offer an alternative bargaining protocol that allows us to extend in a suitable way

\footnotetext{
${ }^{5} \mathrm{~A}$ particular case of games without transferable utility, in which the boundary of the feasible sets is defined by a hyperplane.
} 
each $\alpha$-discounted Shapley value in the TU-games setting to the $\alpha$-consistent value in the NTU-games setting.

The rest of the paper is organized as follows:

Section 2 is devoted to definitions and notation.

Section 3 considers the alternating random proposer bargaining model with the risk of breakdown and time preferences. The equilibrium proposals are characterized and it is proved that the family of discounted Shapley values is parameterized by both, the time cost factor and the probability of breakdown. In the Discussion part we show the differences from the Hart and Mas-Colell approach in considering or not the cost of delay. Additionally, we consider the length in the cost delay explicitly, as in Osborne and Rubinstein (1990), and we show the convergence result when the time between rounds vanishes. Moreover, we use the potential approach to show that each discounted Shapley value can be characterized with efficiency and the property of $\alpha$-balanced contributions. Moreover we show that this value satisfies also the property of $\alpha$-average balanced contributions, which will be useful in the characterization of the $\alpha$-consistent values in the next section.

Section 4 is devoted to games without transferable utility. We consider the scenario where players do not discount payoffs. After rejection, we introduce a probability of $(1-\rho) \alpha$ that the proposer leaves the game and a probability of $(1-\rho)(1-\alpha)$ that the game ends without agreements. The equilibrium proposals are characterized, and we show that TU-games also yield the discounted Shapley values as their average. We also show that, as a result the proposals themselves converge to the average. This fact allows us to characterize the $\alpha$-consistent values in the setting of games without transferable utility in manner that parallels Hart and Mas-Colell (1996) characterization of the consistent values (Maschler and Owen, 1989, 1992).

Section 5 presents the final comments.

\section{Preliminaries}

Let $N=\{1, \ldots, n\}$ be the set of players. A cooperative game with transferable utility (TU-game) is a pair $(N, v)$ where $N$ is a nonempty finite set and $v: 2^{N} \rightarrow \mathbb{R}$ is the characteristic function defined on the power set of $N$ and satisfying $v(\emptyset)=0$. Any element $i$ of $N$ is a player and every nonempty subset $S$ of $N$ is a coalition. The real number $v(S)$ is the worth of the coalition $S$, and it is interpreted as the total payoff that coalition $S$ can obtain for its members independently of the remaining players' behavior. A game $(N, v)$ is monotonic if $v(S) \leq v(T)$ whenever $S \subseteq T$. We will denote with $G^{N}$ the set of all TU-games with player set $N$, and let $\mathbf{G}$ be the set of all games.

Let $(N, v)$ be a TU-game. For each coalition $S$ and each player $i$ in $S$, we call $v(S)-v(S \backslash i)$ the marginal contribution of player $i$ to coalition $S$ in $(N, v)$. The Shapley value of the game $(N, v)$ is the payoff vector $\varphi(N, v) \in \mathbb{R}^{N}$ defined for each $i \in N$ by

$$
\varphi_{i}(N, v)=\sum_{\substack{S \subseteq N \\ i \in S}} \frac{(n-s) !(s-1) !}{n !}[v(S)-v(S \backslash i)],
$$

where $s=|S|$ and $n=|N|$. 
Alternatively, $\varphi(N, v)$ can be obtained recursively ${ }^{6}$ by

$$
\varphi_{i}(S, v)=\frac{1}{s}[v(S)-v(S \backslash i)]+\frac{1}{s} \sum_{j \in S \backslash i} \varphi_{i}(S \backslash j, v), \text { for all } i \in S \subseteq N,
$$

starting with

$$
\varphi_{i}(\{i\}, v)=v(i) \text { for all } i \in N .
$$

The equal division solution is defined by

$$
E_{i}(N, v)=\frac{v(N)}{n}, \text { for all } i \in N
$$

Joosten (1996) introduces two families of solutions with the Shapley value and equal division solution as extreme cases. The first family is called the egalitarian Shapley values, and is obtained as convex combinations of the Shapley value and the equal division solution. For every $\alpha \in[0,1]$, he defines the egalitarian Shapley value $\beta^{\alpha}$ as the solution given by

$$
\beta^{\alpha}(N, v)=\alpha \varphi(N, v)+(1-\alpha) E(N, v) .
$$

The second family $\varphi^{\alpha}, \alpha \in[0,1]$, is defined as

$$
\varphi_{i}^{\alpha}(N, v)=\sum_{\substack{S \subseteq N \\ i \in S}} \frac{(n-s) !(s-1) !}{n !} \alpha^{n-s}[v(S)-\alpha v(S \backslash i)], \quad \text { for all } i \subseteq N .
$$

In Driessen and Radzik (2002) these solutions $\varphi^{\alpha}$ have been called $\alpha$-discounted Shapley values. In van den Brink and Funaki (2010) it is shown that the family $\varphi^{\alpha}$ can be obtained recursively by

$$
\varphi_{i}^{\alpha}(S, v)=\frac{v(S)}{s}+\frac{\alpha}{s}\left[\sum_{j \in S \backslash i} \varphi_{i}^{\alpha}(S \backslash j, v)-v(S \backslash i)\right], \quad \text { for all } i \in S \subset N,
$$

starting with $\varphi_{i}^{\alpha}(\{i\}, v)=v(i)$.

Remark 1 If $|N| \leq 3$, it holds that $\varphi^{\alpha}=\beta^{\alpha}$. For $|N|>3$ there are examples where $\varphi^{\alpha}$ cannot be expressed as a convex combination of the Shapley value and the equal division solution.

\section{Bargaining with risk of breakdown and time preferences}

We show in this section that the family $\varphi^{\alpha}$ can be supported by a bargaining model in which the risk of breakdown and the time cost are the driving forces to reaching an agreement. The time cost preferences in the bargaining process is a factor that should be taken into account by the obvious fact that negotiations take place during that time. Each round requires time, and it is natural to assume that time is costly and that players prefer to reach agreements at the beginning of bargaining.

To model this setting we use alternating offers by a random proposer, as introduced in Hart and Mas-Colell (1996).

The rules are the following:

Let $(N, v) \in G^{N}$ be a TU-game and $0 \leq \rho<1$ be a fixed parameter:

\footnotetext{
${ }^{6}$ See Hart and Mas-Colell (1996).
} 
In each round there is a set $S \subseteq N$ of active players, and a proposer $i \in S$. In the first round, the active set is $S=N$. The proposer is chosen at random from $S$, with all players in $S$ being equally likely to be selected. The proposer makes a feasible offer, i.e. $\sum_{j \in N} a_{j}^{S, i} \leq v(S)$. If all members of $S$ accept the offer -they are asked in some prespecified order- then the game ends with these payoffs. If the offer is rejected by even one member of $S$, then, with probability $\rho$, we move to a next round where the set of active players again is $S$, and, with probability $1-\rho$, breakdown occurs: the proposer $i$ leaves the game, receiving a payoff of zero, and the set of active players becomes $S \backslash i$.

We also assume that players have preferences over the time in which agreements are reached. In particular we suppose that the preferences on $\mathbb{R} \times\{0,1,2,3, .$.$\} can be represented by \delta^{t} x$, that is, for all $t \in\{0,1,2,3, .\},.(x, t) \sim_{i}(y, t+1)$ if and only if $x=\delta y$, where $0 \leq \delta<1$. Moreover, we assume that players are endowed with von Neumann-Morgenstern preferences and are risk-neutral. Therefore, the expected utility of the lottery $\rho x \oplus(1-\rho) y$ is $u_{i}(\rho x \oplus(1-\rho) y)=\rho x+(1-\rho) y$, where the probability of $x$ is $\rho$, and the probability of $y$ is $(1-\rho)$.

The SP equilibrium proposals are characterized in the next proposition.

Proposition 1 Let $(N, v)$ be a monotonic TU-game. Then for each specification of $\rho(0 \leq \rho<1)$ and $\delta(0 \leq \delta \leq 1)$, there is a SP equilibrium. The proposals corresponding to a SP equilibrium are always accepted and they are characterized by the following:

(E.1) $a_{i}^{S, i}(\rho, \delta)=v(S)-\sum_{j \in S \backslash i} a_{j}^{S, i}(\rho, \delta)$ for each $i \in S \subseteq N$; and

(E.2) $a_{j}^{S, i}(\rho, \delta)=\delta\left(\rho a_{j}^{S}(\rho, \delta)+(1-\rho) a_{j}^{S \backslash i}(\rho, \delta)\right)$ for each $i, j \in S$ with $i \neq j$ and each $S \subseteq N$, where $a^{S}(\rho, \delta)=\frac{1}{s} \sum_{i \in S} a^{S, i}(\rho, \delta)$.

Moreover, these proposals are unique and nonnegative.

We use the recursive formula given in the next proposition.

Proposition 2 Let $\left(a^{S}(\rho, \delta)\right)_{S \subseteq N}$ be the average payoff configuration associated with proposals satisfying (E.1) and (E.2), then it holds that

$a_{i}^{S}(\rho, \delta)=\alpha(\rho, \delta)\left[\frac{1}{s}(v(S)-v(S \backslash i))+\frac{1}{s} \sum_{j \in S \backslash i} a_{i}^{S \backslash j}(\rho, \delta)\right]+(1-\alpha(\rho, \delta)) \frac{v(S)}{s}=\varphi_{i}^{\alpha(\rho, \delta)}(S, v), \quad i \in S \subseteq N$,

where $\alpha(\rho, \delta)=\frac{\delta(1-\rho)}{1-\delta \rho}$. Moreover, these vectors $a^{S}(\rho, \delta), S \subseteq N$, are unique and nonnegative.

Proof. Let $(N, v)$ be a monotonic TU-game. By (E.1), for any $i \in S \subseteq N$ we have

$$
s a_{i}^{S}(\rho, \delta)=\left(v(S)-\sum_{j \in S \backslash i} a_{j}^{S, i}(\rho, \delta)\right)+\sum_{j \in S \backslash i} a_{i}^{S, j}(\rho, \delta) .
$$


Applying (E.2),

$$
\begin{aligned}
s a_{i}^{S}(\rho, \delta)= & v(S)-\sum_{j \in S \backslash i} \delta\left(\rho a_{j}^{S}(\rho, \delta)+(1-\rho) a_{j}^{S \backslash i}(\rho, \delta)\right)+\sum_{j \in S \backslash i} \delta\left(\rho a_{i}^{S}(\rho, \delta)+(1-\rho) a_{i}^{S \backslash j}(\rho, \delta)\right) \\
= & v(S)-\delta \rho \sum_{j \in S \backslash i} a_{j}^{S}(\rho, \delta)-\delta \rho a_{i}^{S}(\rho, \delta)-\delta(1-\rho) \sum_{j \in S \backslash i} a_{j}^{S \backslash i}(\rho, \delta)+s \delta \rho a_{i}^{S}(\rho, \delta) \\
& +\delta(1-\rho) \sum_{j \in S \backslash i} a_{i}^{S \backslash j}(\rho, \delta) \\
= & (1-\delta \rho) v(S)-\delta(1-\rho) v(S \backslash i)+s \delta \rho a_{i}^{S}(\rho, \delta)+\delta(1-\rho) \sum_{j \in S \backslash i} a_{i}^{S \backslash j}(\rho, \delta),
\end{aligned}
$$

which finally yields

$$
\begin{aligned}
a_{i}^{S}(\rho, \delta) & =\frac{v(S)}{s}-\frac{1}{s} \frac{\delta(1-\rho)}{1-\delta \rho} v(S \backslash i)+\frac{1}{s} \frac{\delta(1-\rho)}{1-\delta \rho} \sum_{j \in S \backslash i} a_{i}^{S \backslash j}(\rho, \delta) \\
& =\frac{\delta(1-\rho)}{1-\delta \rho}\left[\frac{1}{s}(v(S)-v(S \backslash i))+\frac{1}{s} \sum_{j \in S \backslash i} a_{i}^{S \backslash j}(\rho, \delta)\right]+\left(1-\frac{\delta(1-\rho)}{1-\delta \rho}\right) \frac{v(S)}{s} .
\end{aligned}
$$

The payoffs of the single coalitions $\{i\}$, are $a_{i}^{\{i\}}(\rho, \delta)=v(i)=\varphi_{i}^{\alpha(\rho, \delta)}(\{i\}, v)$, for all $i \in N$. Therefore, the nonnegativity and the uniqueness of $a_{i}^{S}(\rho, \delta)$ follows from the monotonicity of $(N, v)$ and (3) applied recursively.

Finally, comparing (4) with (2), we conclude that $a^{S}(\rho, \delta)=\varphi^{\alpha(\rho, \delta)}(S, v)$, for all $S \subseteq N$.

Proof of Proposition 1. The proof is performed by induction. It is immediate for the 1-player case. Assume it holds when there are less than $n$ players. Let $a^{S, i}(\rho, \delta)$, for $i \in S \subseteq N$, be the proposals of a given SP-equilibrium. Denote with $c^{S}(\rho, \delta)$ the expected payoff vector for the members of $S$ in the subgame where the set of active players is $S$. The induction hypothesis implies that $c^{S}(\rho, \alpha)=a^{S}(\rho, \delta)$ and (E.1), (E.2) are satisfied for $S \neq N$.

Let $d^{N, i}$ be an SP equilibrium proposal vector of player $i \in N$. Among the offers that are accepted, the best move for her is to offer each other player $j \in N \backslash i$ his discounted expected payoff in the case of rejection, namely

$$
d_{j}^{N, i}(\rho, \delta)=\delta\left(\rho c_{j}^{N}(\rho, \delta)+(1-\rho) a_{j}^{N \backslash i}(\rho, \delta)\right),
$$

and then take the entire surplus:

$$
d_{i}^{N, i}(\rho, \delta)=v(N)-\sum_{j \in N \backslash i} \delta\left(\rho c_{j}^{N}(\rho, \delta)+(1-\rho) a_{j}^{N \backslash i}(\rho, \delta)\right)
$$

This proposal is the proposal that is best for $i$ among those that will be accepted if $i$ is the proposer. Moreover,

$$
\begin{aligned}
d_{i}^{N, i}(\rho, \delta) & =v(N)-\delta \rho \sum_{j \in N \backslash i} c_{j}^{N}(\rho, \delta)-\delta \rho c_{i}^{N}(\rho, \delta)-\delta(1-\rho) \sum_{j \in N \backslash i} a_{j}^{N \backslash i}(\rho, \delta)+\delta \rho c_{i}^{N}(\rho, \delta) \\
& =(1-\delta \rho) v(N)-\delta(1-\rho) v(N \backslash i)+\delta \rho c_{i}^{N}(\rho, \delta)
\end{aligned}
$$

and by monotonicity,

$$
(1-\delta \rho) v(N)-\delta(1-\rho) v(N \backslash i) \geq \delta(1-\rho)(v(N)-v(N \backslash i)) \geq 0,
$$


hence $d_{i}^{N, i}(\rho, \delta) \geq \delta \rho c_{i}^{N}(\rho, \delta)$.

On the other hand, any proposal from $i$ that is rejected yields to $i$ at most $\delta \rho c_{i}^{N}(\rho, \delta)$, which is not greater than $d_{i}^{N, i}(\rho, \delta)$. Hence, player $i$ proposes $d^{N, i}=a^{N, i}(\rho, \delta)$ and the proposal will be accepted. From this, it follows that $c^{N}(\rho, \delta)=a^{N}(\rho, \delta)$.

Nonnegativity still must be shown. First, note that the following strategy will guarantee $i$ a nonnegative payoff: as respondent, accept only a payoff of zero, and as proposer, offer zero for every $j \neq i$. This result implies that $a^{N, i}(\rho, \delta)$ must be nonnegative.

Conversely, we show that the proposals $\left(a_{i}^{S, i}(\rho, \delta)\right)_{S \subset N, i \in S}$ satisfying (E.1) and (E.2) do form an SP equilibrium.

We see first that they are all nonnegative. By (3) and monotonicity, it holds that $a_{i}^{S}(\rho, \delta) \geq 0$ for all $i \in S \subseteq N$. This result implies that $a_{j}^{N, i}(\rho, \delta) \geq 0$ for all $j \in N \backslash i$, and that

$$
\begin{aligned}
a_{i}^{N, i}(\rho, \delta) & =(1-\delta \rho) v(N)-\delta(1-\rho) v(N \backslash i)+\delta \rho a_{i}^{N}(\rho, \delta) \\
& \geq \delta(1-\rho)(v(N)-v(N \backslash i))+\delta \rho a_{i}^{N}(\rho, \delta) \geq 0 .
\end{aligned}
$$

We now can verify that the strategies corresponding to these proposals do form an SP equilibrium. By the induction hypothesis, this is so in any subgame with player set $S \neq N$. Fix a player $i \in N$. Given the strategies of the other players, as a proposer, $i$ cannot increase his payoff $a_{i}^{N, i}(\rho, \delta)$ from proposals that are accepted. Making proposals that were systematically rejected, he could only yield the chance to go to the breakdown stage, which provides him an expected payoff of $\delta \rho a_{i}^{N}(\rho, \delta)$. The suggested strategies,however, yield $a_{i}^{N, i}(\rho, \delta)$ which is better than $\delta \rho a_{i}^{N}(\rho, \delta)$. As a responder, $i$ can only deviate by rejecting the offer $a_{i}^{N, j}(\rho, \delta)$ made by another player $j$, but its expectation in the event of continuation, $\delta\left(\rho a_{i}^{N}(\rho, \delta)+(1-\rho) a_{i}^{N \backslash j}(\rho, \delta)\right)$, is only $a_{i}^{N, j}(\rho, \delta)$. The only option that remains is to follow a strategy to drop out of the game, but in this case the payoff is zero and the payoffs associated to the proposed strategies in the SP equilibrium are nonnegative.

Theorem 1 Let $(N, v)$ be a monotonic TU-game. Then for every pair $(\rho, \delta)(0 \leq \rho<1$ and $0 \leq \delta \leq 1)$, there is a unique SP equilibrium. Moreover, for all $i \in S \subseteq N$, we have:

(C.1) The SP equilibrium average payoff vector $a_{i}^{S}(\rho, \delta)$ equals $\varphi_{i}^{\alpha}(S, v)$, the discounted Shapley value of the TU-game $(S, v)$, where $\alpha=\frac{\delta(1-\rho)}{1-\delta \rho}$;

(C.2) For any discount factor $\delta<1$ fixed, when $\rho \rightarrow 1$ it holds that $\left|a_{i}^{S, i}(\rho, \delta)-a_{i}^{S, j}(\rho, \delta)\right| \rightarrow(1-\delta) v(S)$ and $a_{i}^{S}(\rho, \delta) \rightarrow E_{i}(S, v)$; and

(C.3) For any probability of continuation $\rho$ fixed, when $\delta \rightarrow 1$ it holds that $a_{i}^{S}(\rho, \delta) \rightarrow \varphi_{i}(S, v)$, and when $\delta \rightarrow 0$ it holds that $a_{i}^{S}(\rho, \delta) \rightarrow E_{i}(S, v)$.

Proof. (C.1) is an immediate consequence of Propositions (1) and (2). Now, given that $\delta$ is fixed and $\rho \rightarrow 1$, from (E.1) and (E.2) we have

$$
\begin{aligned}
a_{i}^{S, i}(\rho, \delta) & =v(S)-\sum_{j \in S \backslash i} a_{j}^{S, i}(\rho, \delta)= \\
& =v(S)-\sum_{j \in S \backslash i} \delta\left(\rho a_{j}^{S}(\rho, \delta)+(1-\rho) a_{j}^{S \backslash i}(\rho, \delta)\right) \\
& =(1-\delta \rho) v(S)-\delta(1-\rho) v(S \backslash i)+\delta \rho a_{i}^{S}(\rho, \delta),
\end{aligned}
$$


and

$$
a_{i}^{S, j}(\rho, \delta)=\delta\left(\rho a_{i}^{S}(\rho, \delta)+(1-\rho) a_{i}^{S \backslash j}(\rho, \delta)\right),
$$

which implies that $a_{i}^{S, i}(\rho, \delta) \rightarrow(1-\delta) v(S)+\delta a_{i}^{S}(1, \delta)$, and $a_{i}^{S, j}(\rho, \delta) \rightarrow \delta a_{i}^{S}(1, \delta)$.

Moreover,

$$
a_{i}^{S, i}(\rho, \delta)-a_{i}^{S, j}(\rho, \delta)=(1-\delta \rho) v(S)-\delta(1-\rho)\left(v(S \backslash i)+a_{i}^{S \backslash j}(\rho, \delta)\right),
$$

then $\left|a_{i}^{S, i}(\rho, \delta)-a_{i}^{S, j}(\rho, \delta)\right| \rightarrow(1-\delta) v(S)$. From (3) we can write

$$
a_{i}^{S}(\rho, \delta)=\frac{v(S)}{s}+\frac{1}{s} \frac{\delta(1-\rho)}{(1-\delta \rho)}\left[\sum_{j \in S \backslash i} a_{i}^{S \backslash j}(\rho, \delta)-v(S \backslash i)\right],
$$

and then $a_{i}^{S}(\rho, \delta) \rightarrow \frac{v(S)}{s}=E_{i}(S, v)$, so (C.2) follows.

Given $\rho$ fixed, it is also immediate from (5) that (C.3) holds.

It is interesting to compare these convergence results due to the introduction of a discount factor $\delta$ with those of Hart and Mas-Colell without a discount factor:

Without discount factor (H\&MC): for $\delta=1$, when the fear of breakdown vanishes the equilibrium proposals converge to the average, and the average is always the Shapley value (i.e., when $\rho \rightarrow 1$ then $\left.a^{S, i}(\rho, 1) \rightarrow a^{S}(\rho, 1)=S h(S, v)\right)$.

Fixed discount factor: for any $0<\delta<1$ given, when the fear of breakdown vanishes the average equilibrium proposals converge to the equal division solution (i.e., when $\rho \rightarrow 1$ then $\varphi^{\alpha} \rightarrow E$ ). Nevertheless, the proposals do not converge to the average: $\left|a_{i}^{S, i}(\rho, \delta)-a_{i}^{S, j}(\rho, \delta)\right| \rightarrow(1-\delta) v(S)>0$ when $v(S)>0$.

Fixed breakdown risk: for any $0 \leq \rho<1$ given, if the discount factor is close to one then the average equilibrium proposals converge to the Shapley value (i.e., $\delta \rightarrow 1$ then $\varphi^{\alpha} \rightarrow S h$ ). In addition, if the discount factor is close to zero then the average equilibrium proposals converge to the equal division solution (i.e., $\delta \rightarrow 0$ then $\varphi^{\alpha} \rightarrow E$ ).

In summary, the role played by the cost of delay in the Hart and Mas-Colell model and in our model is the same: insofar as the cost of delay vanishes the payoffs converge to the Shapley value. The difference lies in the terminology used. For Hart and Mas-Colell the cost of delay is present in the form of the breakdown probability $(1-\rho)$. In our model, the cost of delay is given by the parameter $\alpha(\rho, \delta)=\frac{\delta(1-\rho)}{(1-\delta \rho)}$, which is a function of the time discount $\delta$ and the breakdown probability $(1-\rho)$ simultaneously.

\subsection{Discussion}

In the seminal works of Sthäl (1972) and Rubinstein (1982) players were endowed with time cost preferences, which is the main force that induces players to reach agreements at the beginning of negotiations in a pure bargaining problem. This setting is given by a pair $(S, d)$, where $S$ is a feasible set, $S \cap \mathbb{R}_{+}^{N} \neq \emptyset$, and $d \in S$ is the disagreement point. The Nash bargaining solution is the point $x \in S$ that maximizes $\left\{\prod_{i \in N}\left(x_{i}-d_{i}\right) \mid x \geq d\right\}$. Binmore (see Binmore and Dasgupta, 1987, chap 4) showed that if the perround discount factor is close to one, then the outcome of the unique subgame perfect equilibrium is 
close to the Nash (1953) bargaining solution. Binmore, Rubinstein and Wolinsky (1986) (see also Roth (1989) subsequently replaced the time preferences (assuming that players are indifferent as to the timing of an agreement) with the risk of breakdown after each proposal's rejection. There, it was proved that when the probability of breakdown converges to zero the equilibrium converges to the Nash bargaining solution. Perhaps due to its technical simplicity, the risk of breakdown was the assumption made in many bargaining models that implement values on coalitional games. That the time discount has been ignored thus far in these models is a bit surprising. A possible answer could be the supposition that, from the implementation point of view, both assumptions were interchangeable. Then, adding both items in the same model becomes redundant. For example, in Hart and Mas-Colell (1996) the following is written:

"Second, we do not consider time discount. The cost of delay in agreement is present in the form of the breakdown probability $\rho$. Time discount would not add anything essential to the analysis. If so desired, however, it could be incorporated with only minor modifications of the conclusions." (Section 2, page 363).

This apparent interchangeability should be presented with caution, as we have observed above. The first scholars to consider simultaneously time preference and the risk of breakdown in the bargaining were Osborne and Rubistein (1990). There, it was shown (see Section 4.5) that when the fear of breakdown rather than the time cost of bargaining is the dominant consideration, the equilibrium is close to the Nash bargaining solution (i.e., $\delta \rightarrow 1$, fixed $\rho$ ), whereas, on the contrary, when the time cost of bargaining rather than the fear of breakdown is the dominant consideration (i.e., $\rho \rightarrow 1$, fixed $\delta$ ), the equilibrium is close to the equal solution (i.e. the point $x \in S$ that maximizes $\left\{\prod_{i \in N} x_{i} \mid x \geq 0\right\}$ ).

Our Theorem 1 can be seen as an extension of the results given in Osborne and Rubistein (1990) into the setting of n-person cooperative TU-games. As in Osborne and Rubinstein, we consider a period in each round as a real time interval of length $\Delta>0$, and examine the limit of the game's SP equilibria when $\Delta$ approaches zero.

As the negotiations take place during the time $\tau \in[0, \infty)$, we assume that each player $i$ has the same probability $p(\tau)$ of being in the game up to time $\tau$. Suppose that the rate at which this probability changes is a proportion of the time, that is, $d p(\tau)=-\omega \tau$. Here, $\omega$ is a positive and constant coefficient of proportionality, fixed by some characteristics of the players. They can be of fitness type, as expected time of life or vitality. Another possibility could be of economic "outside options" type, as in the work salary bargaining, given by the probability of obtaining alternative work in the market. The negative sign means that $p(\tau)$ is decreasing over time. Taking the initial condition $p(0)=1$, the solution of this ordinal differential equation is $p(\tau)=e^{-\omega \tau}$. We enumerate the sequence of rounds by $t=0,1,2, \ldots$, and denote with $\Delta>0$ the length of the delay between rounds. We write $p(t)=e^{-\omega \Delta t}$ as the probability of proposer $i$ being in the game at round $t$. Letting $\gamma=e^{-\Delta}$ we obtain $p(t)=\gamma^{\omega t}$. Under the stationary assumption, the probability of being in the game at round $t$ conditional on still being in the game at round $t-1$ is $\gamma^{\omega}$. When the period of time $\Delta$ of each round approaches zero, $\gamma$ converges to 1 and so $\rho \equiv \gamma^{\omega} \rightarrow 1$. 
On the other hand, when players have preferences over time, insofar as the delay between rounds decreases, the difference in the valuation of the same amount reached at two consecutive rounds decreases. Assume that preferences in time are represented by an exponential discounting function. That is, the present value of $x$ at time $\tau$ is $u(x, \tau)=e^{-r \tau} x$, where $r$ is the parameter that governs the degree of discounting. For example, in financial economics, $r$ is the continuous compounded interest rate. As before we are assuming that a player's attitude toward risk is constant during time and that the players are risk neutral. Therefore, for each round $t, u(x, t)=e^{-r \Delta t} x=\gamma^{r t} x$. In this case the discount factor is $\delta \equiv \gamma^{r}$ and when $\Delta \rightarrow 0$ it holds that $\delta \equiv \gamma^{r} \rightarrow 1$.

Now, given the parameters $(\omega, r)$, the average of the equilibrium proposals is $a(\omega, r)=\varphi^{\alpha(\omega, r)}(N, v)$, where $\alpha(\omega, r)=\frac{\gamma^{r}\left(1-\gamma^{\omega}\right)}{1-\gamma^{r+\omega}}$. Therefore, when the time delay approaches to zero, i.e. $\Delta \rightarrow 0$, it holds that $\gamma \rightarrow 1$, and then

$$
\lim _{\Delta \rightarrow 0} \frac{\gamma^{r}\left(1-\gamma^{\omega}\right)}{1-\gamma^{r+\omega}} \rightarrow \frac{\omega}{\omega+r}
$$

Recall that when $\omega$ decreases the probability $p(t)=e^{-\omega \Delta t}$ of proposer $i$ being in the game at round $t$ increases. Moreover, when $r$ decreases the time cost factor $e^{-r \Delta t}$ increases. Then, we can rephrase the words of Osborne and Rubinstein as follows:

Corollary 1 If the fear of breakdown rather than the time cost of bargaining is the dominant consideration ( $r$ decreases, $\omega$ remain fixed), then the average of the equilibrium proposals converges to the Shapley value $\left(\alpha(\omega, r) \rightarrow 1\right.$ and $\left.\varphi^{\alpha(\omega, r)} \rightarrow S h\right)$. However, if the time cost is the dominant consideration ( $\omega$ decreases, $r$ remain fixed), then the average of the equilibrium proposals converges to the equal division solution $\left(\alpha(\omega, r) \rightarrow 0\right.$ and $\left.\varphi^{\alpha(\omega, r)} \rightarrow E\right)$.

\subsection{Potential and balanced contributions}

In this section we show that $\varphi^{\alpha}$ also has an associated potential function, similar as it was defined in Hart and Mas-Colell (1989).

We first show that $\varphi^{\alpha}$ can be characterized by efficiency and $\alpha$-average balanced contributions.

Proposition 3 Let $(N, v)$ be a TU-game. Then the payoff configuration $\varphi^{\alpha}=\left(\varphi^{\alpha}(S, v)\right)_{S \subseteq N}$ is characterized by

(a) Efficiency: $\sum_{i \in S} \varphi_{i}^{\alpha}(S, v)=v(S)$, for all $S \subseteq N$, and

(b) $\alpha$-Average balanced contributions: $\sum_{j \in S \backslash i}\left(\varphi_{i}^{\alpha}(S, v)-\alpha \varphi_{i}^{\alpha}(S \backslash j, v)\right)=\sum_{j \in S \backslash i}\left(\varphi_{j}^{\alpha}(S, v)-\alpha \varphi_{j}^{\alpha}(S \backslash i, v)\right)$, for all $i \in S$.

Proof. The result is a straightforward application of induction and (2).

Second we show that each $\varphi^{\alpha}$ has an associated $P_{\alpha}$ potential.

Let $\mathbf{G}$ be the set of all games. Given a function $P_{\alpha}: \mathbf{G} \rightarrow \mathbb{R}$ which associates a real number $P_{\alpha}(N, v)$ with every game $(N, v)$, the $\alpha$-marginal contribution of a player $i \in N$ in the game $(N, v)$ is defined by

$$
D^{i} P_{\alpha}(N, v)=P_{\alpha}(N, v)-\alpha P_{\alpha}(N \backslash i, v)
$$


The function $P_{\alpha}$ is an $\alpha$-potential function if it satisfies that $P_{\alpha}(\emptyset, v)=0$ and

$$
\sum_{i \in N} D^{i} P_{\alpha}(N, v)=v(N)
$$

Theorem 2 There exists a unique $\alpha$-potential function $P_{\alpha}$. The $\alpha$-potential of any game $(N, v)$ is uniquely determined by (6) applied only to the game and its subgames. Moreover, $\left.D P_{\alpha}(N, v)=\left(D^{i} P_{\alpha}(N, v)\right)\right)_{i \in N}=$ $\varphi^{\alpha}(N, v)$.

Proof. As $v$ is fixed in what follows, we skip it in the notation. Formula (6) can be rewritten as

$$
P_{\alpha}(N)=\frac{v(N)}{n}-\frac{\alpha}{n} \sum_{i \in N} P_{\alpha}(N \backslash i) .
$$

Starting with $P_{\alpha}(\emptyset)=0, P_{\alpha}(N)$ is determined recursively and uniquely.

Next, we show that $D P_{\alpha}$ satisfy properties (a) and (b) of proposition (3). Property (a) is true by expression (6). To see (b),

$$
\begin{aligned}
\sum_{j \in S \backslash i}\left(D^{i} P_{\alpha}(S)-\alpha D^{i} P_{\alpha}(S \backslash j)\right) & =\sum_{j \in S \backslash i}\left(P_{\alpha}(S)-\alpha P_{\alpha}(S \backslash i)\right)-\alpha \sum_{j \in S \backslash i}\left(P_{\alpha}(S \backslash j)-\alpha P_{\alpha}(S \backslash\{i, j\})\right)= \\
& =\sum_{j \in S \backslash i}\left(P_{\alpha}(S)-\alpha P_{\alpha}(S \backslash j)\right)-\alpha \sum_{j \in S \backslash i}\left(P_{\alpha}(S \backslash i)-\alpha P_{\alpha}(S \backslash\{i, j\})\right)= \\
& =\sum_{j \in S \backslash i}\left(D^{j} P_{\alpha}(S)-D^{j} P_{\alpha}(S \backslash i)\right), \text { for all } i \in S .
\end{aligned}
$$

Then it holds that $D P_{\alpha}$ must coincide with $\varphi^{\alpha}$.

Given $\alpha \in[0,1]$ we say that a solution $\psi$ satisfies $\alpha$-balanced contributions if

$$
\psi_{i}(N, v)-\alpha \psi_{i}(N \backslash j, v)=\psi_{j}(N, v)-\alpha \psi_{j}(N \backslash i, v),
$$

for all $i, j \in N$.

Theorem 3 There exists a unique solution for TU-games that satisfies efficiency and $\alpha$-balanced contributions. This solution is $\varphi^{\alpha}$.

Proof. Existence. As $\varphi^{\alpha}$ satisfies efficiency, it suffices to show that also satisfies $\alpha$-balanced contributions. As we know that $D P_{\alpha}=\varphi^{\alpha}$, we have:

$$
\begin{aligned}
\varphi_{i}^{\alpha}(N)-\alpha \varphi_{i}^{\alpha}(N \backslash j) & =D^{i} P_{\alpha}(N)-\alpha D^{i} P_{\alpha}(N \backslash j)= \\
& =P_{\alpha}(N)-\alpha P_{\alpha}(N \backslash i)-\alpha P_{\alpha}(N \backslash j)+\alpha^{2} P_{\alpha}(N \backslash\{i, j\})= \\
& =D^{j} P_{\alpha}(N)-\alpha D^{j} P_{\alpha}(N \backslash i)=\varphi_{j}^{\alpha}(N)-\alpha \varphi_{j}^{\alpha}(N \backslash i), \text { for all } i, j \in N .
\end{aligned}
$$

Uniqueness. Suppose that there exist two solutions, $\psi$ and $\phi$, that satisfy efficiency and $\alpha$-balanced contributions. By efficiency it holds that $\psi(\{i\})=\phi(\{i\})=v(i)$, for all $i \in N$. Assume by induction that $\psi(S)=\phi(S)$ holds for all $S \subset N, S \neq N$. By $\alpha$-balanced contributions we have that

$$
\psi_{i}(N)-\psi_{j}(N)=\alpha\left(\psi_{i}(N \backslash j)-\psi_{j}(N \backslash i)\right)=\phi_{i}(N)-\phi_{j}(N)
$$

which implies that

$$
\psi_{i}(N)-\phi_{i}(N)=\psi_{j}(N)-\phi_{j}(N) .
$$


As this happens for all pairs $i, j \in N$, there must be a number $d$ such that $\psi_{i}(N)-\phi_{i}(N)=d$, for all $i \in N$. By efficiency, we have that $0=\sum_{i \in N} \psi_{i}(N)-\sum_{i \in N} \phi_{i}(N)=n d$, and so $d=0$. Therefore, we conclude that $\psi(N)=\phi(N)$.

Remark 2 Two different families of efficient potential functions have been considered in Joosten (1996) and in Driessen and Radzik (2002). With the appropriate specification of some of the parameters that defines each family, it can be observed that the $\alpha$-potential function belongs to each of both families.

\section{NTU-games}

We wonder in this section if the previous procedure can yield an efficient solution for the general class of games without transferable utility (NTU-games). In this case, we work with $V(S)$, which is a feasible utility set instead of a number $v(S)$.

We need some additional notation to work with NTU-games.

Given two vectors $x, y \in \mathbb{R}^{N}$, we use the notation $x \cdot y:=\sum_{i \in N} x_{i} y_{i}$, and $x * y:=\left(x_{i} y_{i}\right)_{i \in N}$. If $x \in \mathbb{R}^{N}$ and $\emptyset \neq S \subset N$, we write $x^{S}$ as the restriction of $x$ to $S$, i.e., $x^{S}=\left(x_{i}\right)_{i \in S} \in \mathbb{R}^{S}$. Let $\mathbb{R}_{+}^{N}:=\left\{x \in \mathbb{R}^{N} \mid x \geq 0\right\}$ and $\mathbb{R}_{++}^{N}:=\left\{x \in \mathbb{R}^{N} \mid x>0\right\}$. A set $A \subset \mathbb{R}^{N}$ is called comprehensive if $A-\mathbb{R}_{+}^{N} \subset A$. The boundary of $A$ is denoted by $\partial A$. The boundary is non level if for all $x \in \partial A$ it holds that $\{x\}-\mathbb{R}_{+}^{N} \cap \partial A=\{x\}$. A payoff configuration is a family $\mathbf{x}=\left(x^{S}\right)_{S \subseteq N}$ where $x^{S} \in \mathbb{R}^{S}$ for all $S \subseteq N$. It is efficient if $x^{S} \in \partial V(S)$ for all $S \subseteq N$.

A non-transferable utility game (NTU-game for short), is a map $V$ assigning to each coalition $S$, $\emptyset \neq S \subseteq N$, a subset $V(S) \subset \mathbb{R}^{S}$ of attainable payoff vectors for players in $S$. We assume the same regularity conditions as in Hart and Mas-Colell (1996).

(A.1) for each coalition $S, V(S)$ is non-empty, closed, convex and comprehensive. Moreover, $0 \in V(S)$ and $V_{0}(S):=V(S) \cap \mathbb{R}_{+}^{S}$ is bounded.

(A.2) $\partial V(S)$ is non level and smooth (i.e., for all $x \in \partial A$ there exists a unique ${ }^{7} \lambda=\lambda(x) \in \mathbb{R}_{++}^{S}$ such that $\left.V(S) \subset\left\{y \in \mathbb{R}^{S}: \lambda \cdot y \leq \lambda \cdot x\right\}\right)$.

(A.3) Monotonicity: $V_{0}(S) \times\left\{0^{T \backslash S}\right\} \subset V_{0}(T)$ whenever $S \subset T$.

The assumption (A.3) is just the extension to NTU-games of the classical monotonicity assumption for TU-games. The class of all games that satisfy (A.1), (A.2) and (A.3) is denoted by $\mathcal{G}$.

Two relevant subclasses of NTU-games are:

1. Transferable utility games (TU-games), when for each coalition $S$, there is a number $v(S)$ such that $V(S)=\left\{x \in \mathbb{R}^{S}: \sum_{i \in S} x_{i} \leq v(S)\right\}$ for all $S \subseteq N$. If $V$ is a TU-game, then it will also be denoted by $v$.

2. Hyperplane games (H-games), when $\partial V(S)$ is a hyperplane for all $S \subset N$. That is, for each coalition $S$, there exists a number $v\left(S ; \lambda^{S}\right)$ and a vector $\lambda^{S} \in \mathbb{R}_{++}^{S}$ such that $V(S)=\left\{y \in \mathbb{R}^{S}: \lambda^{S} \cdot y \leq v\left(S ; \lambda^{S}\right)\right\}$. For example, prize games can be modeled in this way: each coalition $S \subseteq N$ has a prize $\pi_{S}$. The prize

\footnotetext{
${ }^{7}$ We normalize it so that $\sum_{i \in S} \lambda^{i}=1$.
} 


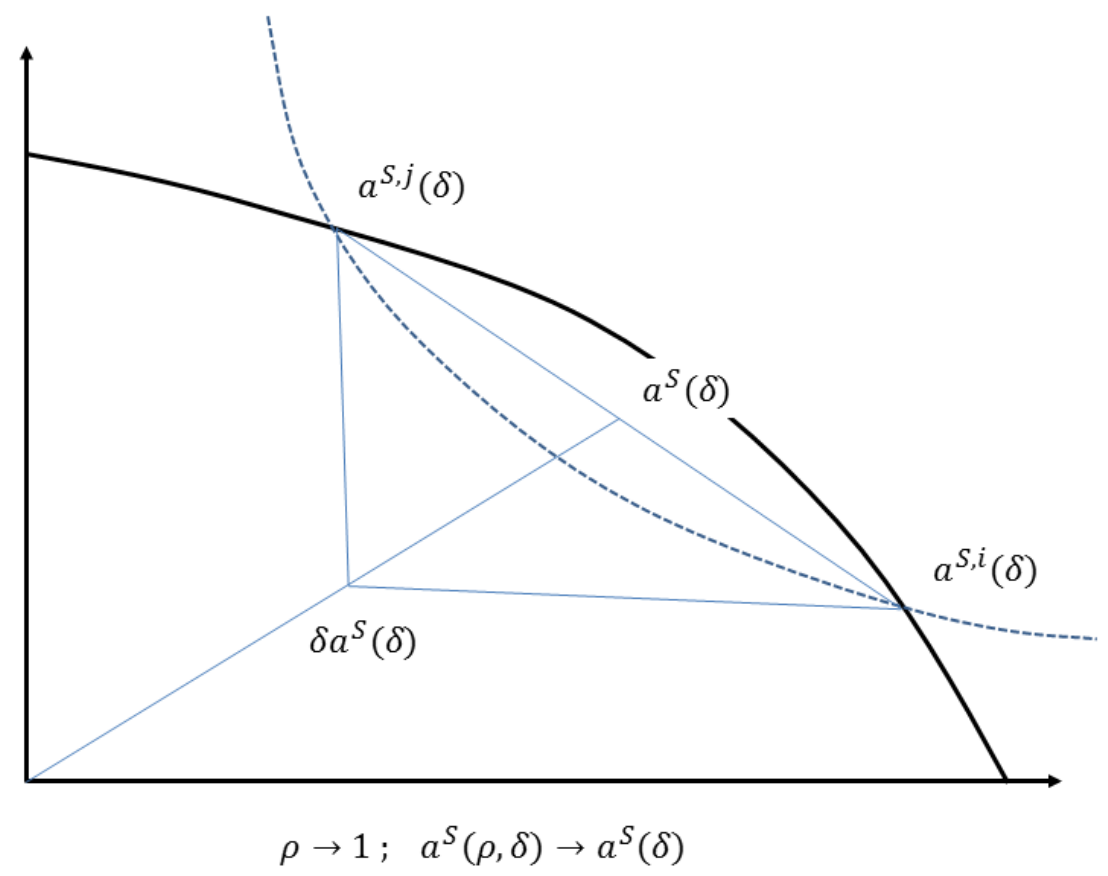

$\pi_{S}$ is indivisible, and only one member of $S$ can receive it. The feasible set of each coalition $S$ consists of all lotteries over which players in $S$ obtain the prize $\pi_{S}$ (for more details see Hart, 1994).

In this setting we can define the bargaining protocol in the same way as before. This protocol only changes the feasibility condition that any proposal must satisfy, which now must be $a^{S, i} \in V(S)$ for any player $i$ in any active coalition $S \subseteq N$.

It is rather straightforward to obtain the characterization of the equilibrium proposals, which we offer in the next proposition.

Proposition 4 Let $(N, V)$ be an NTU-game. Then for each specification of $\rho(0 \leq \rho<1)$ and $\delta(0 \leq$ $\delta \leq 1)$, there is a SP equilibrium. The proposals corresponding to an SP equilibrium are always accepted and they are characterized by:

(E'.1) $a^{S, i}(\rho, \delta) \in \partial V(S)$ for each $i \in S \subseteq N$; and

(E'.2) $a_{j}^{S, i}(\rho, \delta)=\delta\left(\rho a_{j}^{S}(\rho, \delta)+(1-\rho) a_{j}^{S \backslash i}(\rho, \delta)\right)$ for each $i, j \in S$ with $i \neq j$ and each $S \subseteq N$, where $a^{S}(\rho, \delta)=\frac{1}{s} \sum_{i \in S} a^{S, i}(\rho, \delta)$.

Moreover, these proposals are nonnegative.

The problem with this approach is that, in general, $a^{S, i}(\rho, \delta) \neq a^{S, j}(\rho, \delta)$ if $i \neq j$. In addition if $V(S)$ is strictly convex, the average $a^{S}(\rho, \delta)$ might not belong to $\partial V(S)$. Hence, the payoffs need not be efficient. Moreover, given a discount factor $\delta>0$, these proposals could never converge when $\rho \rightarrow 1$. We can see this fact with a simple two players bargaining problem, with $V(\{i\})=V(\{j\})=\mathbb{R}_{-}$. 
To overcome this difficulty we offer an alternative bargaining protocol that for TU-games also implements the discounted Shapley values. However, the equilibrium proposals converge to their average when $\rho \rightarrow 1$. This fact allows us to use hyperplane games in which the equilibrium payoffs are unique. Moreover, under a smoothness assumption on the boundary of the feasible sets, we also my use a convergence result to obtain an efficient payoff configuration.

In our next bargaining model we replace the discount factor with a probability that either the proposer leaves the game, or, the game ends without agreements, in which case breakdown occurs. Hence, in what follows we assume that time is free of cost for players, and so $\delta=1$. The rules are the following.

Let $(N, V)$ be an NTU-game and $0 \leq \rho<1$ and $0 \leq \alpha \leq 1$ be two fixed parameters:

In each round there is a set $S \subseteq N$ of active players, and a proposer $i \in S$. In the first round, the active set is $S=N$. The proposer is chosen at random from $S$, with all players in $S$ being equally likely to be selected. The proposer makes a feasible offer $a^{S, i} \in V(S)$. If all members of $S$ accept the offer -they are asked in some prespecified order- then the game ends with these payoffs. If it is rejected by even one member of $S$, then, with probability $\rho$, we move to a next round where the set of active players is again $S$. With probability $(1-\rho) \alpha$ the proposer $i$ leaves the game, receiving a payoff of zero, and the set of active players becomes $S \backslash i$. And with probability $(1-\rho)(1-\alpha)$ the game ends without agreements, and all players receive a payoff of zero.

This bargaining procedure is a sequential, perfect information game, and it has an SP equilibrium. The proposals associated with the equilibrium are characterized as follows.

Proposition 5 Let $(N, V)$ be an NTU-game. Then for each specification of $\rho(0 \leq \rho<1)$ and $\alpha$ $(0 \leq \alpha \leq 1)$, there is a subgame perfect equilibrium. The proposals corresponding to a subgame perfect equilibrium are always accepted and they are characterized by:

(D.1) $a^{S, i}(\rho, \alpha) \in \partial V(S)$ for all $i \in S \subseteq N$; and

(D.2) $a_{j}^{S, i}(\rho, \alpha)=\rho a_{j}^{S}(\rho, \alpha)+(1-\rho) \alpha a_{j}^{S \backslash i}(\rho, \alpha)$ if $i, j \in S$ with $i \neq j$ and $S \subseteq N$, where $a^{S}(\rho, \alpha)=\frac{1}{s} \sum_{i \in S} a^{S, i}(\rho, \alpha)$ for each $S \subseteq N$.

Moreover, these proposals are nonnegative.

When $\rho \rightarrow 1$, these equilibrium proposals converge to the $\alpha$-consistent values, which are characterized as follows:

Proposition 6 Let $(N, V)$ be an NTU-game and $\boldsymbol{a}(\alpha)=\left(a^{S}(\alpha)\right)_{S \subseteq N}$ a payoff configuration. Then $\boldsymbol{a}(\alpha)$ is an $\alpha$-consistent value if and only if for each $S \subseteq N$ there exists a vector $\lambda_{S} \in \mathbb{R}_{++}^{S}$ such that

(a) $a^{S}(\alpha) \in \partial V(S)$;

(b) $\lambda^{S} \cdot a^{S}(\alpha)=v\left(S ; \lambda^{S}\right):=\max \left\{\lambda^{S} \cdot c: c \in V(S)\right\}$; and

(c) $\sum_{j \in S \backslash i} \lambda_{i}^{S}\left(a_{i}^{S}(\alpha)-\alpha a_{i}^{S \backslash j}(\alpha)\right)=\sum_{j \in S \backslash i} \lambda_{j}^{S}\left(a_{j}^{S}(\alpha)-\alpha a_{j}^{S \backslash i}(\alpha)\right)$, for all $i \in S$.

For hyperplane games the $\alpha$-consistent values are unique, and from (b) and (c) of the above proposition, we have the next recursive formula. 
Proposition 7 Let $\boldsymbol{a}(\alpha)$ be the $\alpha$-consistent value of a hyperplane game, then it holds that

$$
a_{i}^{S}(\alpha)=\frac{1}{s}\left[\sum_{j \in S \backslash i} \alpha a_{i}^{S \backslash j}(\alpha)+\frac{1}{\lambda_{i}^{S}}\left(v\left(S ; \lambda^{S}\right)-\sum_{j \in S \backslash i} \alpha \lambda_{j}^{S} a_{j}^{S \backslash i}\right)\right], \quad i \in S \subseteq N,
$$

starting with $a_{i}^{\{i\}}(\alpha)=v(i):=\max \left\{c_{i}: c_{i} \in V(\{i\})\right\}$.

Note that in the case of TU-games, formula (8) yields

$a_{i}^{S}(\alpha)=\frac{1}{s}\left[\sum_{j \in S \backslash i} \alpha a_{i}^{S \backslash j}(\alpha)+(v(S)-\alpha v(S \backslash i))\right]=\frac{v(S)}{s}+\frac{\alpha}{s}\left[\sum_{j \in S \backslash i} a_{i}^{S \backslash j}(\alpha)-v(S \backslash i)\right], \quad i \in S \subseteq N$,

which is an $\alpha$-discounted Shapley value.

We have the general convergence result:

Theorem 4 Let $(N, V)$ be an NTU-game satisfying assumptions (A.1), (A.2), and (A.3). Then for each $0 \leq \rho<1$ and $0 \leq \alpha \leq 1$ there is an SP equilibrium. Moreover, as $\rho \rightarrow 1$ every limit point of SP equilibrium payoff configuration is an $\alpha$-consistent value payoff configuration of $(N, V)$.

All the proofs of this section are just an straightforward adaptation of the results obtained in Sections 2, 4 and 5, of Hart and Mas-Colell (1996), replacing $a_{i}^{S \backslash j}$ with $\alpha a_{i}^{S \backslash j}$. Therefore, we skip all these proofs.

Remark 3 When we address TU-games, the breakdown stage can be replaced as follows: "with probability $(1-\rho) \alpha$, the proposer $i$ leaves the game, receiving a payoff of zero, and the set of active players becomes $S \backslash i$. With probability $(1-\rho)(1-\alpha)$, the first respondent $j \in S \backslash i$, who has rejected the offer -following a prespecified order ${ }^{8}$ - leaves the game with a payoff of zero, and the set of active players becomes $S \backslash j$ ". In that case, Proposition (5) still holds. To see this, note that if player $j \neq i$ rejects the proposal, his expected payoff in the continuation of the game will be $\rho c_{j}^{N}(\rho, \alpha)+(1-\rho)\left(\alpha a_{j}^{N \backslash i}(\rho, \alpha)+(1-\alpha) 0\right)$. Thus, $d_{j}^{N, i}=\rho c_{j}^{N}(\rho, \alpha)+(1-\rho)\left(\alpha a_{j}^{N \backslash i}(\rho, \alpha)\right)$, for $j \neq i$, and $d_{i}^{N, i}=v(N)-\sum_{j \neq i} d_{j}^{N, i}$, is the proposal that is best for $i$ among the proposals that will be accepted if $i$ is the proposer. Moreover,

$$
\begin{aligned}
d_{i}^{N, i} & =v(N)-\sum_{j \neq i} d_{j}^{N, i}= \\
& =v(N)-\rho \sum_{j \neq i} c_{j}^{N}(\rho, \alpha)-\rho c_{i}^{N}(\rho, \alpha)-(1-\rho) \alpha \sum_{j \neq i} a_{j}^{N \backslash i}(\rho, \alpha)+\rho c_{i}^{N}(\rho, \alpha) \\
& =(1-\rho)(v(N)-\alpha v(N \backslash i))+\rho c_{i}^{N}(\rho, \alpha) .
\end{aligned}
$$

On the other hand, any proposal from $i$ that is rejected yields to $i$ at most

$$
\rho c_{i}^{N}(\rho, \alpha)+(1-\rho)\left(\alpha 0+(1-\alpha) \max \left\{a_{i}^{N \backslash j}(\rho, \alpha) \mid j \neq i\right\}\right) .
$$

By monotonicity and an induction argument, for all $j \neq i$ it holds that $a_{i}^{N \backslash j}(\rho, \alpha) \leq v(N \backslash j) \leq v(N)$, and then

$$
\begin{gathered}
(1-\alpha) \max \left\{a_{i}^{N \backslash j}(\rho, \alpha) \mid j \neq i\right\} \leq(1-\alpha) v(N) \\
=v(N)-\alpha v(N) \leq v(N)-\alpha v(N \backslash i) .
\end{gathered}
$$

\footnotetext{
${ }^{8}$ The order in which the respondents are asked does not affect the result of the bargaining.
} 
Hence,

$$
\rho c_{i}^{N}(\rho, \alpha)+(1-\rho)(1-\alpha) \max \left\{a_{i}^{N \backslash j}(\rho, \alpha) \mid j \neq i\right\} \leq \rho c_{i}^{N}(\rho, \alpha)+(1-\rho)(v(N)-\alpha v(N \backslash i))=d_{i}^{N, i} .
$$

Then player $i$ proposes $d^{N, i}=a^{N, i}(\rho, \alpha)$ and the proposal will be accepted. From this, it follows that $c^{N}(\rho, \alpha)=a^{N}(\rho, \alpha)$.

It is an open problem whether a similar argument can be reproduced for the NTU-games case. Although we have not found counterexamples, the proof of the characterization of the equilibrium proposals with this breakdown variation is not immediate.

\section{Conclusion}

The two families of the discounted and the egalitarian Shapley values can be compared either axiomatically or strategically. Our claim is that the axiomatic comparison is not conclusive. We briefly review the axiomatic approach.

In van den Brink, Funaki and Ju (2011) it is shown that all egalitarian Shapley values are characterized by Sobolev reduced game consistency (Sobolev, 1973), and $\alpha$-standardness for two-player games. In Joosten (1996) it is shown that the discounted Shapley values are characterized by Hart and Mas-Colell reduced game consistency (Hart and Mas-Colell, 1989), and $\alpha$-standardness. Such an $\alpha$-standardness axiom requires that in a two-player game, both players obtain a fraction $\alpha \in[0,1]$ of their singleton worth, and the remainder is split equally among them. More formally,

- A solution $\psi$ satisfies $\alpha$-standardness for two player games, $\alpha \in[0,1]$, if for every game $(N, v)$ with $N=\{i, j\}, i \neq j$, it holds that $\psi_{i}(N, v)=\alpha v(i)+\frac{1}{2}(v(N)-\alpha(v(i)+v(j)))$.

In this way the difference lies in the type of consistency considered: either with the Sobolev reduced game, or the Hart and Mas-Colell reduced game. Their definitions are:

- A solution $\psi$ satisfies Sobolev consistency if for every $(N, v)$ with $n \geq 2, j \in N$, and $x=\psi(N, v)$, it holds that $\psi_{i}\left(N \backslash j, v^{x}\right)=\psi_{i}(N, v)$ for all $i \in N \backslash j$, where the reduced game $\left(N \backslash j, v^{x}\right)$ is given by

$$
v^{x}(S)=\frac{s}{n-1}\left(v\left(S \cup j-x_{j}\right)+\frac{n-1-s}{n-1} v(S), \text { for all } S \subseteq N \backslash j .\right.
$$

- A solution $\psi$ satisfies Hart and Mas-Colell consistency if for every $(N, v)$ with $n \geq 2, T \subset N$, and all $i \in N$, it holds that $\psi_{i}\left(T, v_{T}^{\psi}\right)=\psi_{i}(N, v)$, where the reduced game $\left(T, v_{T}^{\psi}\right)$ is given by

$$
v_{T}^{\psi}(S)=\left(v\left(S \cup T^{c}\right)-\sum_{j \in T^{c}} \psi_{j}\left(S \cup T^{c}\right), \text { for all } S \subseteq T \text { with } T^{c}=N \backslash T .\right.
$$

Both definitions have in common an internal consistency of the solution when some players leave the game. However, apart from this fact, the definitions of consistency are rather different and they do not permit a clear comparison between them.

Another axiomatic comparison is possible with the use of the properties of $\alpha$-egalitarianism and $\alpha$ reducing player properties. A player is said to be a null player in a game $(N, v)$ if all of her marginal 
contributions are zero. In Joosten (1996) (see also van den Brink, Funaki and Ju, 2011) it is shown that each $\alpha$-egalitarian Shapley value satisfies the property of $\alpha$-egalitarian null player property:

- A solution $\psi$ is $\alpha$-egalitarian if for every null player $i \in N$ in a game $(N, v)$ it holds that $\psi_{i}(N, v)=$ $(1-\alpha) \frac{v(N)}{n}$.

When $\alpha=1$, this is just the null player property. Moreover, each $\alpha$-egalitarian Shapley value $\beta^{\alpha}$, $\alpha \in[0,1]$, is characterized by the properties of efficiency, symmetry, linearity and $\alpha$-egalitarianism.

In van den Brink and Funaki (2010) it is shown that each $\alpha$-discounted Shapley value satisfies the property of $\alpha$-reducing player property. A player $i \in N$ in a game $(N, v)$ is an $\alpha$-reducing player if $v(S \cup i)=\alpha v(S)$.

- A solution $\psi$ satisfies the $\alpha$-reducing player property if $\psi_{i}(N, v)=0$ when $i$ is an $\alpha$-reducing player in $(N, v)$.

Again, when $\alpha=1$, this property corresponds to the null player property. Moreover, each $\alpha$-discounted Shapley value $\varphi^{\alpha}, \alpha \in[0,1]$, is characterized by efficiency, symmetry, linearity and $\alpha$-reducing player properties.

Both axiomatic systems differ only in the $\alpha$-egalitarianism and $\alpha$-reducing player properties. Again, however, the comparison between both is not conclusive. They look equally natural, artificial, or both

In this paper we have considered the discounted Shapley values from a strategic point of view.

The bargaining model considered is based on the alternating random-order proposer, where there is a probability $(1-\rho)$ of a breakdown in the negotiations in the case that the proposal is rejected by some respondent. In the case that breakdown occurs, we have considered two different options:

In the first option, only the proposer leaves the game (as in the original model of Hart and Mas-Colell, 1996). However, we assume additionally a positive cost of delay in the payoffs given by a time discount $\delta$. In the second option, there is a probability $\alpha$ that the proposer leaves the game, and $(1-\alpha)$ that the game ends without agreements.

In both cases we have observed that the average of the SP equilibria payoffs is a $\varphi^{\alpha}$ value. In both models the degree of egalitarianism in the payoffs is given by the same factor: the relative bargaining power between the proposer and the respondent. With more power the proposer is better able to fully extract her expected marginal contributions in the game, and, hence, closer are the payoffs to the Shapley value. On the contrary, when the power moves to the respondent, the expected marginal contributions of the proposer associated with the ultimatum threats of leaving the game are taken into account to a lesser extend and, hence, the asymmetries between players given by their differences in their marginal contributions disappear. This fact yields outcomes closer to the equal division solution.

The source of this bargaining power is different in both models. In the first model, only the proposer has a chance to make threats by leaving the game after a rejection. From this fact, it looks as if she is the only player who has the power of commitment in the bargaining. Nevertheless, the time cost factor $\delta$ provides the respondent with bargaining power, because rejecting the offer forces the finding of the agreement at least in the next round, lowering the proposer's expected payoffs by the discount factor. 
Therefore, the higher cost of delay implies higher bargaining power for the respondent, and the outcome will be more egalitarian.

In the second model, the parameter $\alpha /(1-\alpha)$ measures the relative power of commitment, because it is the quotient of the probability that the proposer leaves the game if any offer she makes is rejected, $(1-\rho) \alpha$, and the probability that the game ends if her offer is rejected, $(1-\rho)(1-\alpha)$. The relative distance between the Shapley value and the equal division solution depends of this relative bargaining power even if the absolute level of power of commitment is quite low $(\rho \rightarrow 1)$.

The main conclusion that we can extract from both models is that, insofar as the bargaining power is shared in some way between the proposer and the respondents in the negotiations it should be natural to consider outcomes between the Shapley value and the equal division solution. The agreements will be more or less close to such solutions depending of the relative bargaining power that the proposer and the respondents have in their bilateral face-to-face interaction. There are two different families between both extremes: the egalitarian Shapley values (a convex combination between them) and the discounted Shapley values. In the TU-games setting, both families can be obtained as equilibrium payoffs of a bargaining process: either as a variation of the bidding for the surplus mechanism or as a variation of the alternating random proposer mechanism. As in both cases time is used in each round, it is natural to assume that time is costly for players, which implies that they prefer not to delay the agreements. We have observed that in both type of models the introduction of a time discount factor automatically produces as a result a discounted Shapley value. From this point of view the family of discounted Shapley values seems more natural than the family of egalitarian Shapley values.

\section{Acknowledgments}

We wish to thank Francesc Carreras for several comments and suggestions. Emilio Calvo thanks the Ministry of Science and Technology, the European Feder Funds under project ECO2010-20584, and the Generalitat Valenciana under the Excellence Programs Prometeo 2009/068 and ISIC2012/021 for their financial support. Esther Gutiérrez-López wishes to thank financial support from the Spanish Ministry of Science and Technology and the European Regional Development Funds under project ECO2012-33618, and from the Universidad del País Vasco / Euskal Herriko Unibertsitatea (UFI 11/51).

\section{References}

Binmore, K., \& Dasgupta, P. (1987). The Economics of Bargaining. Oxford. Blackwell.

Binmore, K., Rubinstein, A., \& Wolinsky, A. (1986). The Nash Bargaining Solution in Economic Modelling. The RAND Journal of Economics 17, 176-188.

Driessen, T.H., \& Radzik, T. (2002): "A weighted pseudo-potential approach to values fot TU-games," International Transactions in Operational Research 9, 303-320. 
Hart, S (1994). On Prize Games. In Essays in Game Theory, ed. by N. Meggido. Springer-Verlag. New York, 111-121.

Hart, S., \& Mas-Colell, A. (1989). Potential, Value, and Consistency. Econometrica 57, 589-614.

Hart, S., \& Mas-Colell, A. (1996). Bargaining and Value. Econometrica 64, 357-380.

Joosten, R. (1996). Dynamics, equilibria and values dissertation. Maastricht University.

Ju, Y \& Wettstein, D. (2009) Implementing cooperative solution concepts: a generalized bidding approach. Economic Theory 39, 307-330.

Maschler, M., $\&$ Owen, G. (1989). The Consistent Shapley Value for Hyperplane Games. International Journal of Game Theory 18, 389-407.

Maschler, M., \& Owen, G. (1992). The Consistent Shapley Value for Games without Side Payments. In Rational Interaction, ed. by R. Selten. Springer-Verlag. New York, 5-12.

Myerson, R.B. (1980). Conference Structures and Fair Allocation Rules. International Journal of Game Theory 9, 169-182.

Nash, J.F. (1953). Two-Person Cooperative Games. Econometrica 21, 128-140.

Osborne, M., 6 Rubinstein, A. (1990). Bargaining and Markets. San Diego: Academic Press.

Pérez-Castrillo, D., $\&$ Wettstein, D. (2001). Bidding for the Surplus: a Non-Cooperative Approach to the Shapley Value. Journal of Economic Theory 100, 274-294.

Roth, A. (1989). Risk Aversion and the Relationship between Nash's Solution and Subgame Perfect Equilibrium of Sequential bargaining. Journal of Risk and Uncertainty 2, 353-365.

Rubinstein, A. (1982). Perfect Equilibrium in a Bargaining Model. Econometrica 50:97-109.

Shapley, L.S. (1953). A Value for n-Person Games. In Contributions to the theory of Games II (Annals of Mathematics Studies 28), ed. by H. W. Kuhn, $\&$ Tucker, A.W. Princeton University Press. Princeton, 307-317.

Sobolev, A.I. (1973). A Characterization of Optimality Principles in Cooperative Games by Functional Equations (Russian). Mathematical Methods of Social Sciences 6, 94-151.

Stähl, I. (1972). Bargaining Theory. Stockholm: Stockholm School of Economics

van den Brink, R., \& Funaki, Y. (2010). Axiomatization and Implementation of Discounted Shapley Values. Tinbergen Institute Discussion Paper, 10-065/1.

van den Brink, R., Funaki, Y. \& Ju, Y. (2011) Reconciling marginalism with egalitarianism: consistency, monotonicity, and implementation of egalitarian Shapley values. Social Choice and Welfare DOI 10.1007/s00355-011-0634-2. 
Young, P. (1985). Monotonic solutions for cooperative games. International Journal of Game Theory $14,65-72$. 